\title{
Ileal Conduit for Reconstruction of the Duodenum following Right Hemicolectomy and Pancreaticoduodenectomy for Malignant Duodenocolic Fistula
}

\author{
Capt. A KOhri \\ $B M, R A M C(V)$ *
}

MrSKOhri

FRCS, FRCSEd**

\section{*Oxford University Officers' Training Corps, Yeomanry House, Manor Road, Oxford, OXI $3 U Q$. \\ **Department of Surgery, Hammersmith Hospital, The Royal Postgraduate Medical School, Du Cane Road, London.}

SUMMARY: We describe a case of malignant duodenocolic fistula in which after a radical resection of right $\vec{\circ}$ hemicolectomy and pancreaticoduodenectomy, the duodenal defect was reconstructed using a conduit of transposed $\rightarrow$ ileum. This technique, not previously described, offers an alternative to 'duodenal patching', which allows only limited $\vec{\omega}$ duodenal resection, or gastroenterostomy with its attendant side-effects such as dumping syndrome.

\section{Introduction}

Malignant duodenocolic fistulae are rare, with a reported incidence of 1 in 900 colorectal carcinomas (1). Bypass procedures of gastrojejunostomy, ileotransverse colostomy and proximal transverse jejunostomy have been described(2), but provide poor palliation. Wide en bloc resection provides the only hope of cure and was first described in 1944 involving extended right hemicolectomy with pancreaticoduodenectomy as a two stage procedure (3). This was later modified by Janes and Mills to a one stage procedure (4). Alternatively, a less radical palliative resection may be undertaken involving a right hemicolectomy with excision of a disc of duodenal wall. In 18 cases described, this procedure was associated with a high post-operative mortality (5). This has been attributed to a frequent incidence of duodenal dehiscence and fistula formation encountered when closing the duodenum with a primary suture technique. The mucosal patch technique has been successfully reported in one case (5) and was first described for the repair of contused anterior duodenal wall following blunt trauma (6). Alternatively a serosal patch may be employed using the serosal surface of a convenient loop of small bowel (7).

Following curative resection of extended right hemicolectomy with pancreaticoduodenectomy we describe the reconstruction of the duodenum using a conduit of transposed ileum, a technique not previously described. This technique obviates the need for a gastrojejunostomy and would seem to be more physiologically desirable.

\section{Clinical Details}

The patient, a 78-year-old female, presented with diarrhoea, weight loss and vomiting. She was cachectic and weighed $48 \mathrm{Kg}$. Barium studies and endoscopy revealed a carcinoma of the hepatic flexure extending to involve the third part of the duodenum. Ultrasono-

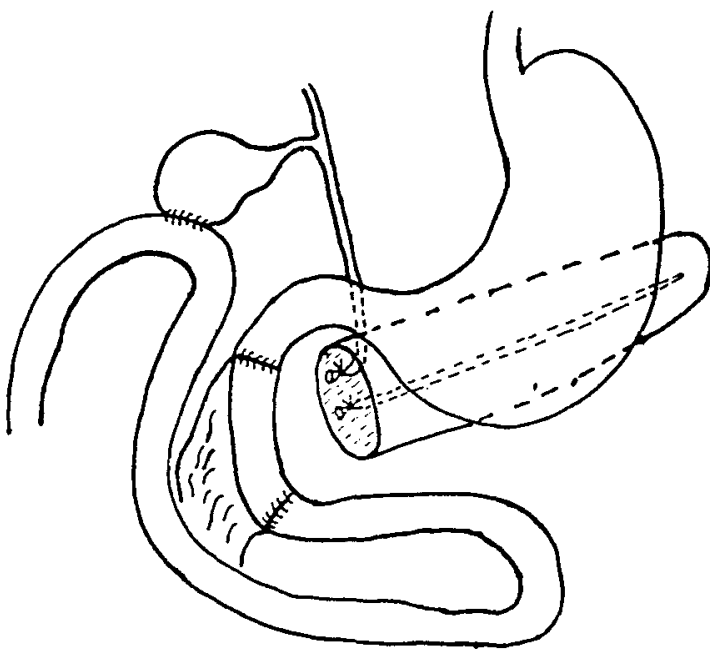

Fig 1. Diagramatic representation of ileal conduit for reconstruction of the duodenum, following right hemicolectomy and pancreaticoduodenectomy.

graphy revealed no evidence of liver metastases. She was initially parenterally fed to improve her nutritional status for surgery.

\section{Technique}

A standard right hemicolectomy was performed with full mobilisation of the entire second and third parts of 0 the duodenum and head of pancreas. The second and

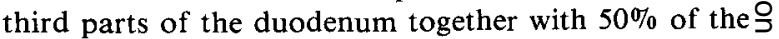
head of the pancreas was excised en bloc with the right $>$ hemicolectomy specimen. The open ends of the common bile duct and pancreatic duct were ligated. The cut edge $N$ of the remaining pancreas were oversewn to achieve haemostasis. A segment of terminal ileum to match the duodenal defect was mobilised with the mesentry as an 
ileal conduit, and anastomosed to the two ends of cut duodenum. To achieve biliary drainage a standard cholecystojejenostomy was carried out (Fig 1). No attempt was made to drain the pancreatic duct, leaving it tied off. A tube drain was left down to the head of the pancreas and the abdomen closed.

\section{Post-operative Course}

Parenteral nutrition was continued in the post-operative period, which was complicated by the development of a pancreatic fistula with the appearance of amylase rich fluid in the drain. This was managed conservatively and dried up by the 30th post-operative day. A subsequent gastrograffin swallow was performed to check the integrity of the ileal conduit. Oral fluids were introduced following by diet and pancreatic enzyme supplements. After a prolonged period of slow mobilisation due to her poor nutritional status she was allowed home seven weeks post-operatively. Histology confirmed adenocarcinoma of the hepatic flexure (Dukes B) with all resection margins clear of tumour.

\section{Discussion}

This technique avoids formal pancreatic resection and pancreaticoenterostomy, a formidable procedure in an ill and malnourished patient. We believe restoring duodenal continuity with an ileal conduit is more physiological than gastroenterostomy with its associated morbidity such as dumping syndrome. Experience of pancreatic ligation in segmental pancreatic transplantation in the animal model has shown a degree of long term endocrine impairment (8), but there is little clinical data to support this. We feel the avoidance of a hazardous pancreatico-enteric anastomosis in an ill patient is justified, although in a nutritionally superior patient this may be a viable option at the time of surgery, avoiding the subsequent risk of pancreatic fistulation. Owing to the rarity of this condition we must await further cases before we can justify with results this technique as another surgical option in the management of malignant duodenocolic fistulae.

\section{REFERENCES}

1. Welch J P, Warshan A L. Malignant duodenocolic fistulas. Am J Surg 1977; 133: 658-61.

2. Gallager H W. Extended right hemicolectomy: the treatment of advanced carcinoma of the hepatic flexure and malignant duodenocolic fistual. $B r J$ Surg 1980; 47: 616-21.

3. Linton R R. Two stage operation for carcinoma of transverse colon producing duodenocolic fistula: a report of two cases. Arch Surg 1944; 48: 197-207.

4. JANes R M, Mills J R. Malignant duodenocolic fistula - report of a case treated successfully by mass resection. Con J Surg 1959; 3: 91-2.

5. Cawthorn S J, Bett N J, Rutter K R P. Malignant duodenocolic fistua: long-term survival following an extended right hemicolectomy with wide local excision of the duodenum. B J Surg 1984; 72: 211 .

6. Austin Jones S, Joergensen E J. Closure o duodenal wall defects. Surgery 1963; 53: 438-42.

7. Ellis H, Naunton-Morgan M, Wastell C. Cur ative surgery in carcinoma of the colon involving duodenum. Br J Surg 1972; 59: 932-5.

8. INGRAM N P. MS. Thesis University of London 1986. 